\title{
Perfil del Sabor del Clon CCN51 del Cacao (Theobroma cacao L.) Producido en Tres Fincas del Municipio de San Vicente de Chucurí
}

\author{
Flavor Profile of Clone CCN 51 Cocoa (Theobroma \\ cacao L.) Produced in Three Farms Municipality of \\ San Vicente de Chucurí
}

Quintana Fuentes Lucas Fernando', Goméz Castelblanco Salomón²

${ }^{1}$ Escuela de Ciencias Básicas, Tecnología e Ingeniería, Universidad Nacional Abierta y a Distancia. UNAD, Bucara-

manga, Colombia, lucas . quintana1@unad. edu.co

2 Universidad Nacional Abierta y a Distancia., UNAD, Bucaramanga, Colombia, salomon. gomez@unad. edu. co

\section{Resumen}

Colombia es uno de los mayores productores de cacao en América, su producción es consumida en su totalidad en forma interna. El departamento de Santander es el mayor productor de cacao en Colombia, y el Municipio de San Vicente de Chucurí es considerado la capital cacaotera del país. Actualmente la Federación Colombiana de Cacaoteros (Fedecacao), junto con la UNAD tiene un convenio de cooperación para el fortalecimiento de la capacidad evaluadora de la calidad sensorial del cacao.

En el proceso de evaluación de la calidad sensorial del licor de cacao (clon CCN 51) se establecieron las necesidades de acuerdo a la normatividad vigente para la implementación de un panel de jueces entrenados, desde la preselección, selección, entrenamiento específico, evaluación del entrenamiento y evaluación de las muestras finales de tres fincas representativas en donde se cultiva el clon CCN 51 del municipio de San Vicente de Chucurí.

Se conformó un grupo de 11 jueces y se obtuvo el perfil sensorial de sabor para las muestrasade tres fincas representativas del municipio.

Palabras clave: licor de cacao, clon CCN51, perfil sensorial

\section{Abstract}

Colombia is one of the bigger producers of cocoa in America, the production is consumed in the totally in internal form. The department of Santander is me bigger producer of cocoa in Colombia and the municipality of San Vicente de Chucuri is considered the cocoa capital of the country. Actually the Colombian federation of cocoa (Fedecacao) together with the Unad have an agreement of cooperation for the strengthening of the evaluating capacity of the sensorial quality of the cocoa.

In the process of evaluation of the sensorial quality of the cocoa liqueur (clone CNN 51) we established the needs in accordance to the current regulations for the implementation of a panel of trained judges, from the preselection, selection, specific training, training evaluation and the evaluation of the final samples of three representative farms in where is cultivated the clone CCN 51 in San Vicente del Chucuri.

We conform a group of eleven trained judges and we obtained the sensorial profile of flavor for the samples for three representative farms of the municipality. 


\section{Introducción}

El presente artículo es producto del proyecto de investigación cuyo objetivo era establecer el "Perfil sensorial del cacao producido en San Vicente de Chucuri", el cual se realizó en convenio con Fedecacao y la Universidad Nacional Abierta y a Distancia - UNAD.

Fedecacao ha realizado diferentes estudios sobre la calidad físico-química y nutricional del cacao, pero no ha profundizado en el análisis sensorial, siendo este el aspecto tratado en el desarrollo de la investigación. La evaluación sensorial se define como el método científico utilizado para evocar, medir, analizar e interpretar aquellas respuestas percibidas a través de los sentidos de la vista, gusto, olfato, tacto y oído [1].

En la actualidad no es suficiente caracterizar un alimento desde un punto de vista fisicoquímico, microbiológico y nutricional, sino que, si se quiere tener éxito en el mercado nacional y una posible expansión hacía mercados internacionales, hay que considerar la calidad sensorial del producto como un factor crítico para el acceso a nuevos mercados competitivos.

En principio, la caracterización sensorial y su correlación con las características fisicoquímicas de los alimento, son necesarias para definir lo que se entiende por el perfil sensorial, para determinar si logra o no satisfacer las demandas del consumidor, así como para conocer cuáles son los atributos sensoriales que más influyen en su aceptabilidad. Una vez conformado el grupo de jueces se logró caracterizar sensorialmente el cacao suministrado por Fedecacao y producido en tres fincas representativas con una confiabilidad del $99 \%$ teniendo en cuenta lo establecido en la caracterización de los productores realizada por Corpoica en 2000 , donde se determinó que corresponde a un cacao tipo forastero con predominio del sabor a cacao, ácido, astringente y amargo.

\section{Generalidades}

La zona productora de cacao se encuentra localizada en la Montaña Santandereana,Vertiente Occidental de la Cordillera Oriental, que recorre el departamento de sur a norte, bordeando el valle del río Magdalena. En esta subregión, se encuentran localizados, Landázuri, El Carmen, San Vicente, Rionegro, El Playón y Lebrija (Figura 1) municipios, importantes por el área de explotación dedicho sistema de producción [2].

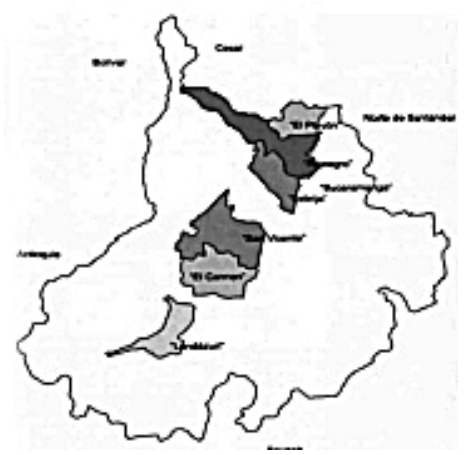

Fuente: IGAC, Corpoica (2000)

Figura 1. Distribución municipios productores de cacao de Santander

La tipificación de los productores se realizó en el nivel municipal teniendo en cuenta sus particularidades y diferencias estadísticas entre los mismos, además de la utilidad práctica de los resultados para las acciones de transferencia de tecnología y de asistencia técnica.

Con base en el diagnóstico de los productores de cacao de Santander desarrollado por la corporación de investigaciones en ciencias agropecuarias Corpoica y a los tres grupos conformados de acuerdo a las características referentes a al área total de la finca, porcentaje de cacao híbrido, sanidad del cultivo, asistencia técnica, administración directa del cultivo, densidad de siembra, rendimientos, distancia a la cabecera municipal y a la experiencia de la unidad técnica de Fedecacao-Fondo Nacional del Cacao del municipio de San Vicente de Chucuri se seleccionó una finca de cada uno de los grupos de productores de cacao clonado y en especial el CCN51. 


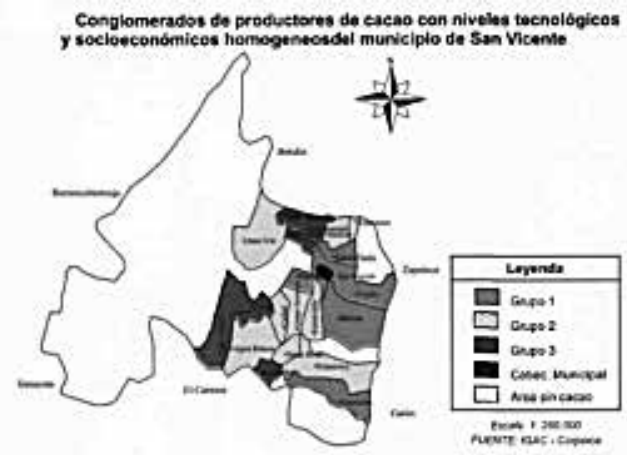

Fuente: Corpoica. (2000)

Figura 2. Distribución de grupos productores de cacao en San Vicente de Chucurí

De un grupo conformado por los productores con fincas en las veredas de Santa Inés, Centro, Mérida, el Guamal, Palmar y el Naranjo ubicadas a una altura entre 700 y 1100 msnm, fincas cercanas al municipio, con mayores facilidades de acceso y en donde predomina el arreglo de cacao con maderables, cítricos y con cultivo de plátano. Se escogió la finca Chimita de la vereda Centro.

Un segundo grupo de productores están en las veredas de Santa Rosa, Aguablanca, La Granada, La Esperanza, Ceibal, La Esmeralda, Primavera, Campo Hermoso, Esmeralda Alta, Llana Fría y Los Medios, ubicadas entre los 550 hasta 1100 msnm predomina el arreglo de cacao con aguacate, plátano (dominico y banano) y árboles maderables como el cedro y móncoro. La finca el Seúl de la vereda Santa Rosa fue la seleccionada.

Un tercer grupo lo conforman las veredas de Pertrecho, Filón, Alto Viento, León, Marcito, Cantarranas, Barro Amarillo y Palestina. El arreglo predominante es el de cacao con aguacate, maderables y plátano hartón. De éste grupo se selecciono la finca El Consuelo, de la vereda Barro Amarillo.

En San Vicente se reportan 2367 fincas con un área de cultivo tradicional de 11066,4 hectáreas, de las cuales 896,8 corresponden a material clonado y una producción de 8346 Toneladas. Aunque en los 24 municipios donde se realizó el censo cacaotero de 2007, la mayoría de plantaciones corresponden a material híbrido, hay que tener en cuenta que las 5.316,4 hectáreas sembradas con clones, son hectáreas relativamente nuevas. En la grafica 1, se observa que el clon objeto de estudio es uno de los dos clones más sembrados en la región. El CCN-51 es un cacao clonado de origen ecuatoriano de alta productividad.

El clon CCN51 fue seleccionado y estudiado por Homero Castro hace más de 30 años, investigó la población de cacao del alto Amazonas del Ecuador, coleccionando material genético para usarlos en programas de cruzamiento con VariedadesTrinitarias y otros cultivos, buscando un clon de alta calidad y gran productividad resistente a las enfermedades. Este clon es el resultado del cruce de los clones ICS 95 y el IMC 67 en 1965 en la zona de Naranjal en la hacienda "Sofi", se han realizado diversos análisis del grano de cacao del CCN51, comparándolo con el cacao de las huertas tradicionales, con excelentes resultados [3].

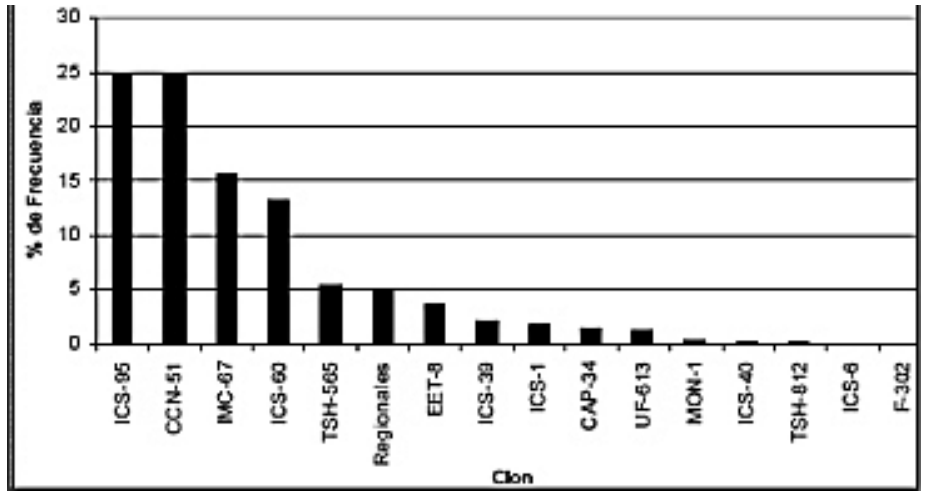

Fuente: Censo cacaotero en Santander Rincón-Guarín, (2007).

Figura 3. Frecuencia de producción por clon en Santander. 


\section{Metodología}

\section{Generalidades selección de jueces}

La Guía Técnica Colombiana 165 nos presenta este proceso como la base para la ejecución adecuada de la evaluación sensorial, en ella se establece el tipo de juez que debemos tener para garantizar la aplicación adecuada de la prueba escogida; en nuestro caso debemos considerar un tipo de juez experto o entrenado para poder dar respuesta a la prueba descriptiva que aplicamos [4].

De acuerdo con lo establecido por Sancho [5], el flujograma del proceso de selección inicia con la preselección, selección de precandidatos, pruebas de selección, entrenamiento especifico, evaluación del entrenamiento especifico y análisis de muestras problema [5].

En cada una de estas etapas se aplicó lo establecido en el manual del Instituto de Ciencia y Tecnología de Alimentos (ICTA), de la Universidad Nacional de Colombia [6]. Donde se establecen las siguientes pruebas: sabores básicos, identificación de aromas, intensidad de color, pruebas de pares [7], dúo -trío [8] y triangular [9].

Con estas pruebas se busca evaluar la capacidad de cada uno de los candidatos y llevar a cabo la selección del personal. Cada una de estas pruebas sigue un protocolo de aplicación que se ajusta al siguiente flujo: preparación de la muestra, codificación de los vasos, acondicionamiento de cabinas, convocatoria de los jueces, realización de la prueba y análisis de resultados [10].

\section{Condiciones de prueba}

Las condiciones para el desarrollo y aplicación de las diferentes pruebas sensoriales, es necesario proporcionar las condiciones locativas básicas, para la sala de catación o cabina, y para el sitio de preparación de las muestras. También se tiene un especial cuidado en el momento de elegir la prueba que se va a aplicar, el formulario, el número de muestras, las cantidades, los alimentos adicionales que van a servir de vehículo para ingerir la muestra y los recipientes que van a contener las muestras. Lo anterior, brinda la seguridad y confiabilidad de los resultados, para que a través del estudio estadístico, lograr un análisis significativo [11], [12].

El desarrollo de las pruebas se debe llevar a cabo en un lugar que cumpla con unas condiciones que favorezcan unos resultados eficientes; debe disponer de una infraestructura adecuada, poseer un instrumental y personal calificado. En la mayoría de los casos se deben garantizar las condiciones para poder emitir un juicio en forma individual [13], [11], [14].

\section{Preparación de muestras}

Las muestras de licor de cacao se prepararon según lo establecido en el manual para análisis de cacao [15], [16]. Es importante resaltar que las muestras de licor fueron procesadas y suministradas por Fedecacao en San Vicente de Chucurí para efectuar el análisis sensorial.

Los pasos establecidos son:

1. Tostado de las almendra. (NTC 1252, ICONTEC, 2003).

2. Descascarillado.

3. Triturado de los cotiledones tostados.

4. Refinado de la mezcla obtenida hasta el tamaño de partícula deseado.

5. Moldeado y refrigerado.

6. Atemperado en baño maría al momento de ser servidas para el análisis sensorial.

\section{Perfil de sabor}

El método seleccionado debido al grado de entrenamiento de los jueces fue el consenso que consiste en: inicialmente, el evaluador trabaja solo, registrando las características o notas de sabor. Tan pronto se tienen las notas de sabor, se recomienda consensuarlas. Con base en las notas definidas, se determina 
el orden de percepción, intensidad, sabor residual o persistencia, o ambos, y luego se evalúa la impresión total [17].

Jiménez (2003), señala que en un licor de cacao se pueden identificar tres tipos de sabores: básicos, específicos y adquiridos, los cuales se detallan a continuación [18], [19].

\section{Sabores básicos}

Acido: describe licores con sabor ácido, debido a la presencia de ácidos volátiles y no volátiles, se percibe a los lados y centro de la lengua.

Amargo: se describe como un sabor fuerte y amargo, generalmente por la falta de fermentación, se percibe en la parte posterior de la lengua o en la garganta.

Dulce: se percibe en la punta de la lengua.

Astringente: este sabor fuerte es por la falta de fermentación, provoca sequedad en la boca, aumento de salivación, se percibe en toda la boca, lengua, garganta y hasta en los dientes,

\section{Sabores específicos}

Cacao: describe el sabor típico a granos de cacao bien fermentados, secos, asados y libres de defectos. Floral: presentan sabores a flores, casi perfumados posiblemente se perciba un olor como a químico.

Frutal: se caracteriza por licores con sabor a fruta madura. Esto describe una nota de aroma a dulce, agradable.

Nuez: se relaciona con el sabor de la almendra y nuez.

\section{Sabores adquiridos}

Moho: describe licores con sabor mohoso, debido a una sobre fermentación de las almendras o a un incorrecto secado.

Químico: describe licores contaminados por combustible, plaguicidas, desinfectante y otros productos químicos.

Verde/crudo: se describe así generalmente por la falta de fermentación o de tostado.

Humo: describe licores contaminados por humo de madera, usualmente debido al secado artificial.

En la NTC 3929 se establece una escala para la evaluación de la intensidad de cada uno de los atributos descritos anteriormente que va de 0 a 5 pero que en nuestro caso del cacao se ha ajustado a la que utilizan los laboratorios en Ecuador, Francia, Venezuela y Colombia que va de 0 a 10, y que nos describen cada uno de los atributos de la siguiente manera:

$0-0=$ Ausente

1-2 = Intensidad baja

3-5 = Intensidad media

6-8 = Intensidad alta

9-10 = Intensidad muy alta/fuerte

\section{Análisis de datos}

Existen dos enfoques básicos para el manejo de los datos. En los métodos de perfil por consenso, inmediatamente después de que los panelistas han completado sus evaluaciones, el líder del panel tabula los resultados e inicia una discusión para resolver las diferencia [20]. En otros métodos de análisis descriptivo no hay discusión y el perfil obtenido es una serie de promedios de los puntajes asignados a 
cada descriptor por cada panelista. Los promedios se pueden comparar estadísticamente; por ejemplo, usando el análisis de varianza. También existen técnicas de análisis multivariado, para todos los métodos de análisis descriptivo [17], [21].

\section{Análisis de varianza. "ANOVA"}

El análisis de varianza es una técnica que nos permite estudiar si hay diferencias significativas entre las medias de las calificaciones asignadas a más de dos muestras, basándonos en el principio de $t$-Students [22]. Podemos aplicar la técnica para explicar el desarrollo del comportamiento de los datos del proceso de evaluación del licor de cacao por parte de los jueces. En nuestro caso la aplicamos en dos niveles [23].

1. Una vía, donde se explica la diferencia entre una variable del estudio.

2. Dos vías, donde se explica la diferencia entre dos variables del estudio.

Análisis de componentes principales. "ACP" El análisis de componentes principales tiene este objetivo: dada en observaciones de $p$ variables, se analiza si es posible representar adecuadamente esta información con un número menor de variables construidas como combinaciones lineales de las originales [24].

El análisis de componentes principales puede resumir la mayor parte de las variables obtenidas en un análisis sensorial a dos o tres de mayor importancia que nos permitirán, al final, diferenciar el licor de cacao y poder relacionar sus característica [25].

\section{Análisis de Resultados}

\section{Evaluación de licores en fincas seleccionada}

Con estos resultados se tooó la decisión de proseguir y someter las muestras de CCN 51 a juicio de los 11 jueces expertos en entrenamiento. Las muestras de licor de cacao fueron procesadas de acuerdo a los protocolos establecidos y suministradas por Fedecacao en San Vicente de Chucuri, siendo sometidas a la evaluación por parte del grupo de jueces.

El formato utilizado para la evaluación sensorial de los licores es el aplicado por el laboratorio de investigación de calidad de cacao y café del Instituto Autónomo de Investigaciones Agropecuarias (INIAP) del Ecuador.

Antes de proceder a determinar los perfiles de sabor para cada una de las muestras, se evaluó el comportamiento de los jueces realizando la prueba de ANOVA en una sola vía para los resultados de cada muestra problema y se estableció que no hay diferencia significativa para las medias de los datos generados por cada juez con un nivel de significancia del $1 \%$.

Con los resultados del análisis sensorial para cada una de las muestras de licor de cacao de cada una de las fincas (tabla 3), se realiza la prueba de análisis de componentes principales, en la tabla 4 , la matriz de correlación obtenida indica que no hay diferencias significativas entre ellas por el índice de correlación obtenido superior a $(0,7)$. 
Tabla 2. Descripción muestras licores CCN51

\begin{tabular}{|c|c|c|}
\hline \multirow{6}{*}{$\begin{array}{l}\text { Muestra } 1 \\
\text { Código } 410\end{array}$} & Finca & El consuelo \\
\hline & Propietario & Luis Eduardo León Vásquez \\
\hline & Vereda & Barro Amarillo \\
\hline & Altura & 900 msnm \\
\hline & Tiempo de fermentación & 144 horas \\
\hline & \% de fermentación & 70 \\
\hline \multirow{6}{*}{$\begin{array}{l}\text { Muestra } 2 \\
\text { Código } 012\end{array}$} & Finca & Seúl \\
\hline & Propietario & Isaías León \\
\hline & Vereda & Santa Rosa \\
\hline & Altura & $830 \mathrm{msnm}$ \\
\hline & Tiempo de fermentación & 144 horas \\
\hline & \% de fermentación & 60 \\
\hline \multirow{6}{*}{$\begin{array}{l}\text { Muestra } 3 \\
\text { Código } 804\end{array}$} & Finca & Chimita \\
\hline & Propietario & Manuel Acevedo \\
\hline & Vereda & Centro \\
\hline & Altura & $980 \mathrm{msnm}$ \\
\hline & Tiempo de fermentación & 144 horas \\
\hline & \% de fermentación & 87 \\
\hline
\end{tabular}

Fuente: elaboración propia

Tabla 3. Resultado análisis sensorial licores de cacao por finca.

\begin{tabular}{|l|c|c|c||}
\hline \multicolumn{1}{|c|}{ Cydigo } & Sebl & El consuelo & Chimita \\
\hline Cacao & 2,3 & 2,7 & 3,7 \\
\hline Acidez & 5 & 4,6 & 2,2 \\
\hline Astringencia & 3,6 & 3,4 & 2,9 \\
\hline Amargor & 4,7 & 4,2 & 3,2 \\
\hline Frutal & 0,3 & 0,5 & 0,8 \\
\hline Floral & 0,6 & 0 & 0,2 \\
\hline Nuez & 0,9 & 0,8 & 1,6 \\
\hline Verde & 0,4 & 0,4 & 0,2 \\
\hline Otros & 0,4 & 0 & 0 \\
\hline \hline
\end{tabular}

Fuente: elaboración propia 
Tabla 4. Matriz de Pearson licores de cacao fincas.

\begin{tabular}{|l|c|c|c|}
\hline \multicolumn{1}{|c|}{ Variables } & Sebl & El Consuelo & Chimita \\
\hline Seúl & 1 & 0,985 & 0,769 \\
\hline El Consuelo & 0,985 & 1 & 0,840 \\
\hline Chimita & 0,769 & 0,840 & 1 \\
\hline
\end{tabular}

Fuente: elaboración propia

Lo anterior indica que hay consistencia en los resultados obtenidos; se observó un índice de correlación positiva en el rango de 0,769 a 0,985.

Adicionalmente, se establece la ubicación de los licores por finca con respecto a las características sensoriales evaluadas y así establecer el perfil de cada una de ellas, como puede observarse en las figuras 4 y 5 .

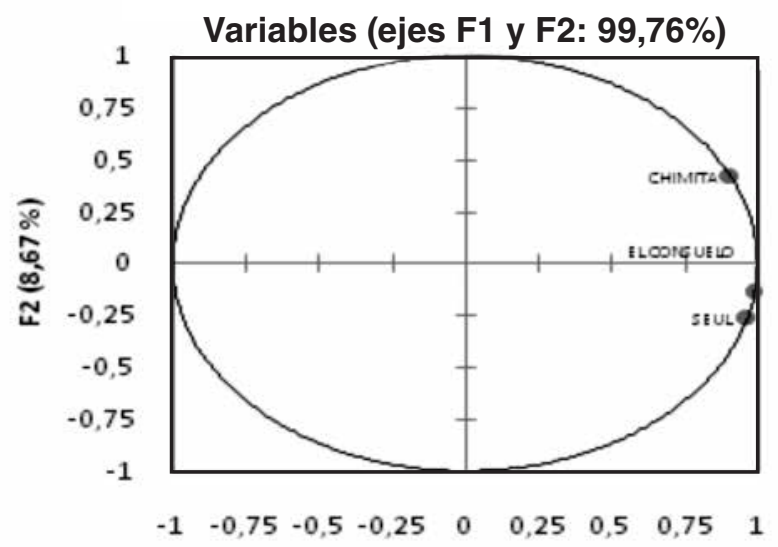

Fuente: elaboración propia

Figura 4. Comportamiento licores de cacao fincas

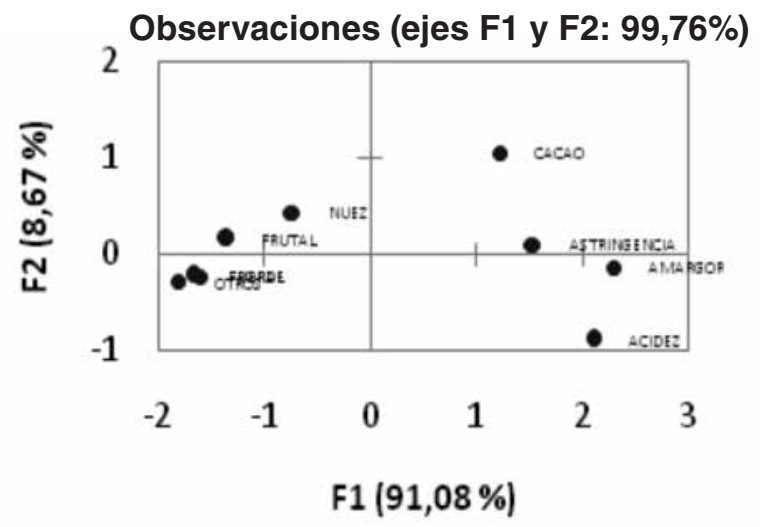

Fuente: elaboración propia

Figura 5. Comportamiento atributos licores de cacao fincas 


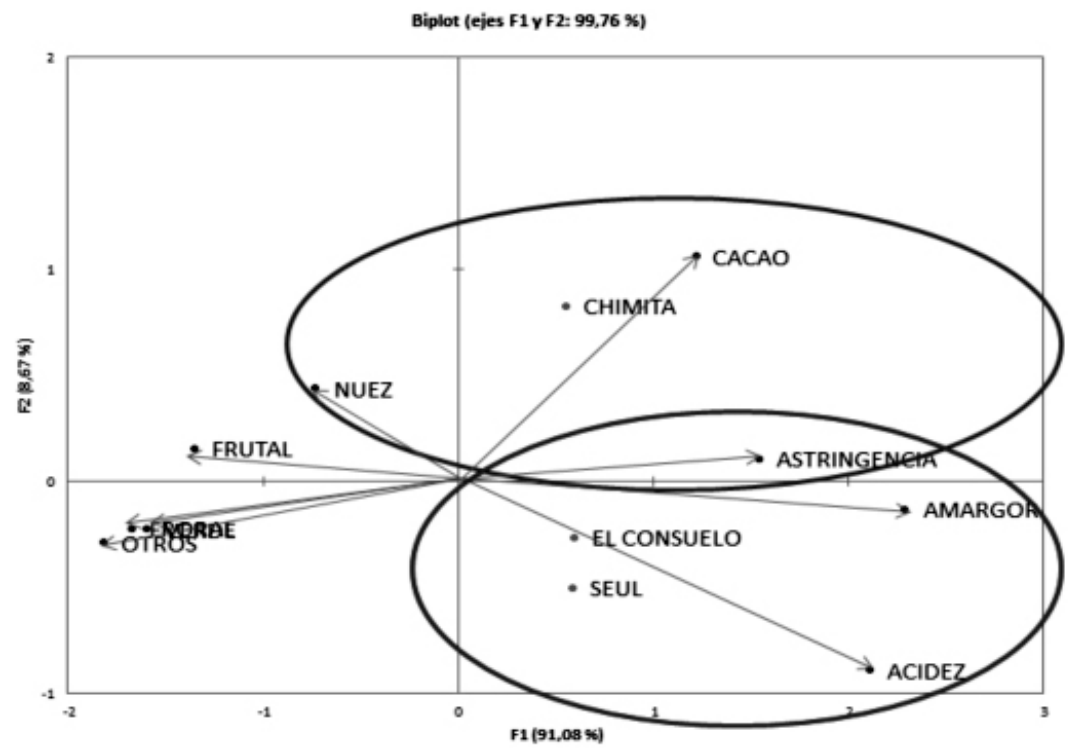

Fuente: elaboración propia

Figura 6. Comportamiento licores vs. atributos

Según la figura 4, observamos que la muestra 3 (correspondiente a la finca Chimita) presenta una mayor intensidad en los atributos sabor a cacao y astringencia, con una menor correlación con los atributos amargo y ácido, y en una correlación negativa con los atributos adquiridos de sabor a nuez, floral, frutal, caramelo. Las muestras 1 y 2 de la finca Seúl y el Consuelo presentan mayor correlación con los atributos amargo y acido, en menor grado con astringente y cacao, y en forma inversa con los adquiridos en igual forma que la muestra de la finca Chimita.

Según [17], el perfil arrrojado por el calculo de promedios en cada característica corresponde a los resultados contenidos en la tabla 4 y representados en la grafica 4, como el perfil sensorial de los licores de cada una de las fincas y el perfil correspondiente para cada una de las veredas (figuras 6, 7 y 8).

Tabla 4. Resultados análisis de licores.

\begin{tabular}{|l|l|l|l|l|l|l|l|l|l|l||}
\hline ORIGEN & CODIGO & CACAO & ACIDEZ & ASTRINGENCIA & AMARGOR & FRUTAL & FLORAL & NUEZ & VERDE & OTROS \\
\hline $\begin{array}{l}\text { Seul } \\
\text { (santa } \\
\text { rosa) }\end{array}$ & 12 & 2,3 & 5 & 3,6 & 4,7 & 0,3 & 0,6 & 0,9 & 0,4 & 0,4 \\
\hline $\begin{array}{l}\text { Consuelo } \\
\text { (barro } \\
\text { amarillo) }\end{array}$ & 410 & 2,7 & 4,6 & 3,4 & 4,2 & 0,5 & 0 & 0,8 & 0,4 & 0 \\
\hline $\begin{array}{l}\text { Chimita } \\
\text { (centro) }\end{array}$ & 804 & 3,7 & 2,2 & 2,9 & 3,2 & 0,8 & 0,2 & 1,6 & 0,2 & 0 \\
\hline
\end{tabular}

Fuente: elaboración propia 


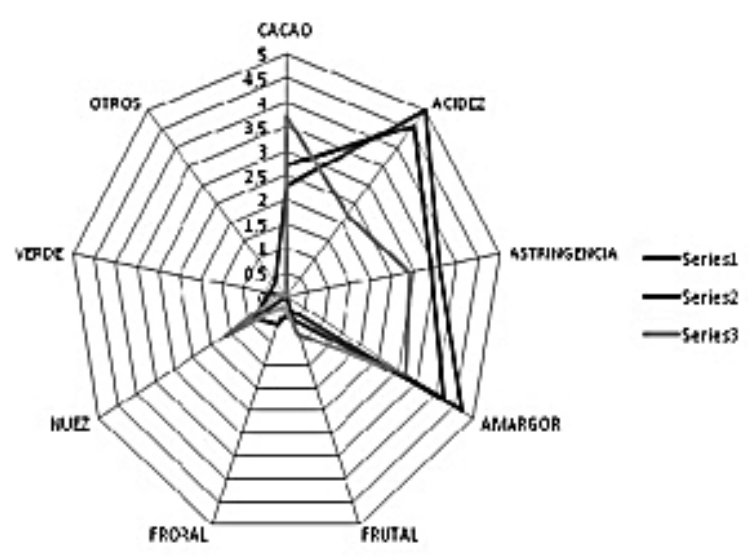

Fuente: elaboración propia

Figura 5. Perfil sensorial de los licores evaluados por finca.

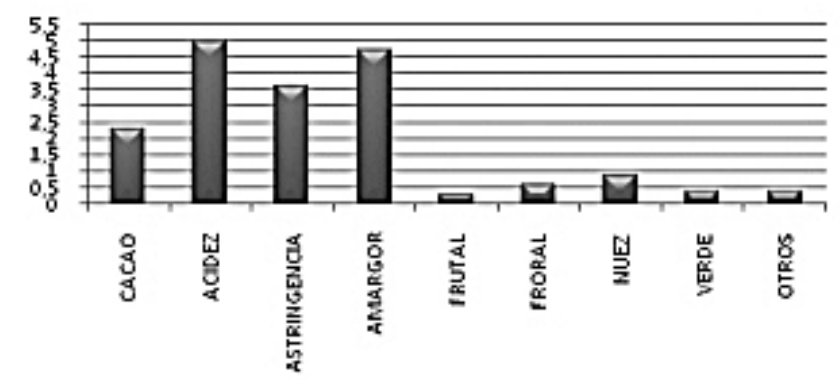

Fuente: los autores

Figura 6. Perfil sensorial Finca Seúl, vereda Santa Rosa

Tabla 5. Análisis de varianza finca Seúl

\begin{tabular}{|l|c|r|c|c|c|c||}
\hline \begin{tabular}{|} 
Origen de las \\
variaciones
\end{tabular} & $\begin{array}{c}\text { Suma de } \\
\text { cuadrados }\end{array}$ & $\begin{array}{c}\text { Grados de } \\
\text { libertad }\end{array}$ & $\begin{array}{c}\text { Prome- } \\
\text { dio de los } \\
\text { cuadrados }\end{array}$ & F & Probabilidad & $\begin{array}{c}\text { Valor crutico } \\
\text { para F }\end{array}$ \\
\hline Entre grupos & 11,0666667 & 9 & 1,22962963 & 0,22829637 & 0,989491652 & 1,999114806 \\
\hline $\begin{array}{l}\text { Dentro de los } \\
\text { grupos }\end{array}$ & 430,888889 & 80 & 5,386111111 & & & \\
\hline Total & 441,955556 & 89 & & & & \\
\hline & Nivel de & Tabla F & $\begin{array}{c}\text { Compara- } \\
\text { tivo }\end{array}$ & $\begin{array}{c}\text { Valor F } \\
\text { calculado }\end{array}$ & $\begin{array}{c}\text { Diferencia } \\
\text { significativa }\end{array}$ & \\
\hline \multirow{2}{*}{ sueces } & 0,05 & 1,99911481 & $>$ & 0,22829637 & NO & \\
\cline { 2 - 8 } & 0,01 & 2,63739843 & $>$ & 0,22829637 & NO & \\
\hline
\end{tabular}

Se observa que los datos son consistentes en un nivel de significancia superior a 95\% de confiabilidad.

Fuente: elaboración propia 


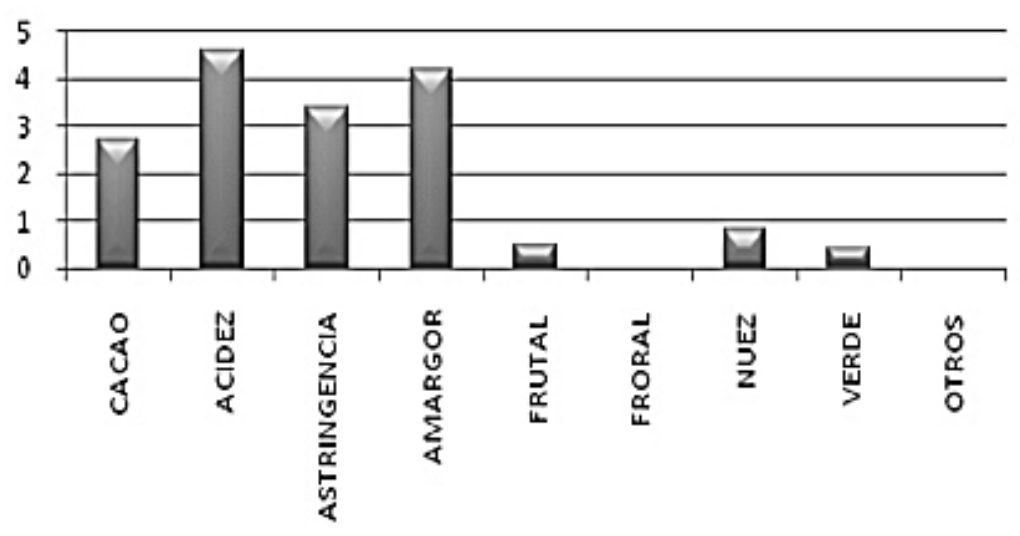

Fuente: los autores.

Figura 7. Perfil sensorial Finca Consuelo, vereda Barro Amarillo

Tabla 6. Análisis de varianza finca Consuelo

\begin{tabular}{|l|c|r|c|c|c|c||}
\hline $\begin{array}{c}\text { Origen de } \\
\text { las } \\
\text { variaciones }\end{array}$ & $\begin{array}{c}\text { Suma } \\
\text { de cuadra- } \\
\text { dos }\end{array}$ & $\begin{array}{c}\text { Grados de } \\
\text { libertad }\end{array}$ & $\begin{array}{c}\text { Prome- } \\
\text { dio de los } \\
\text { cuadrados }\end{array}$ & F & Probabilidad & $\begin{array}{c}\text { Valorcrutico } \\
\text { para F }\end{array}$ \\
\hline Entre grupos & 12,71111111 & 9 & 1,412345679 & 0,29803309 & 0,973316415 & 1,999114806 \\
\hline $\begin{array}{l}\text { Dentro de los } \\
\text { grupos }\end{array}$ & 379,1111111 & 80 & 4,738888889 & & & \\
\hline Total & 391,8222222 & 89 & & & & \\
\hline & $\begin{array}{c}\text { Nivel } \\
\text { de signifi- } \\
\text { cancia }\end{array}$ & Tabla F & $\begin{array}{c}\text { Compara- } \\
\text { tivo }\end{array}$ & $\begin{array}{c}\text { Valor F } \\
\text { calculado }\end{array}$ & $\begin{array}{c}\text { Diferencia } \\
\text { significativa }\end{array}$ & \\
\hline \multirow{2}{*}{ Jueces } & 0,05 & 1,99911481 & $>$ & 0,29803309 & NO & \\
\cline { 2 - 8 } & 0,01 & 2,63739843 & $>$ & 0,29803309 & NO & \\
\hline
\end{tabular}

Se observa que los datos son consistentes en un nivel de significancia superior a $95 \%$ de confiabilidad. Fuente: los autores.

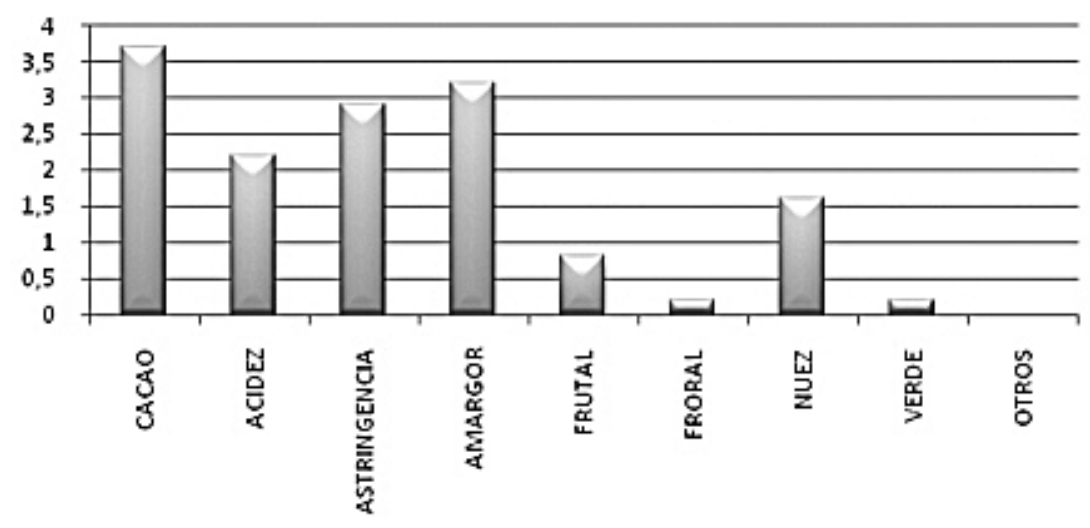

Fuente: elaboración propia

Figura 8. Perfil sensorial de la Finca Chimita, vereda Centro 
Tabla 7. Análisis de varianza finca Chimita

\begin{tabular}{|c|c|c|c|c|c|c|}
\hline $\begin{array}{c}\text { Origen } \\
\text { de las } \\
\text { variaciones }\end{array}$ & $\begin{array}{c}\text { Suma de } \\
\text { cuadrados }\end{array}$ & $\begin{array}{c}\text { Grados de } \\
\text { libertad }\end{array}$ & $\begin{array}{c}\text { Promedio } \\
\text { de los } \\
\text { cuadrados }\end{array}$ & F & $\begin{array}{c}\text { Probabili- } \\
\text { dad }\end{array}$ & $\begin{array}{c}\text { Valor crutico } \\
\text { para F }\end{array}$ \\
\hline $\begin{array}{c}\text { Entre gru- } \\
\text { pos }\end{array}$ & 13,9555556 & 9 & 1,550617284 & 0,47957236 & 0,884309765 & 1,999114806 \\
\hline $\begin{array}{c}\text { Dentro de } \\
\text { los grupos }\end{array}$ & 258,666667 & 80 & 3,233333333 & & & \\
\hline Total & 272,622222 & 89 & & & & \\
\hline & $\begin{array}{c}\text { Nivel de } \\
\text { significan- } \\
\text { cia }\end{array}$ & Tabla F & Comparativo & $\begin{array}{c}\text { Valor F } \\
\text { calculado }\end{array}$ & $\begin{array}{c}\text { Diferencia } \\
\text { significativa }\end{array}$ & \\
\hline \multirow{2}{*}{ Jueces } & 0,05 & 1,99911481 & $>$ & 0,47957236 & No & \\
\cline { 2 - 7 } & 0,01 & 2,63739843 & $>$ & 0,47957236 & No & \\
\hline
\end{tabular}

Se observa que los datos son consistentes en un nivel de significancia superior a $95 \%$ de confiabilidad.

\section{Fuente: elaboración propia}

Con estos resultados, y verificando los porcentajes de fermentación inicial de las muestras, se puede inferir que los perfiles establecidos son válidos y nos dan un diagnóstico inicial de la calidad sensorial del cacao para el clon CCN51 seleccionado como el más representativo y promisorio para el municipio de San Vicente de Chucurí (S).

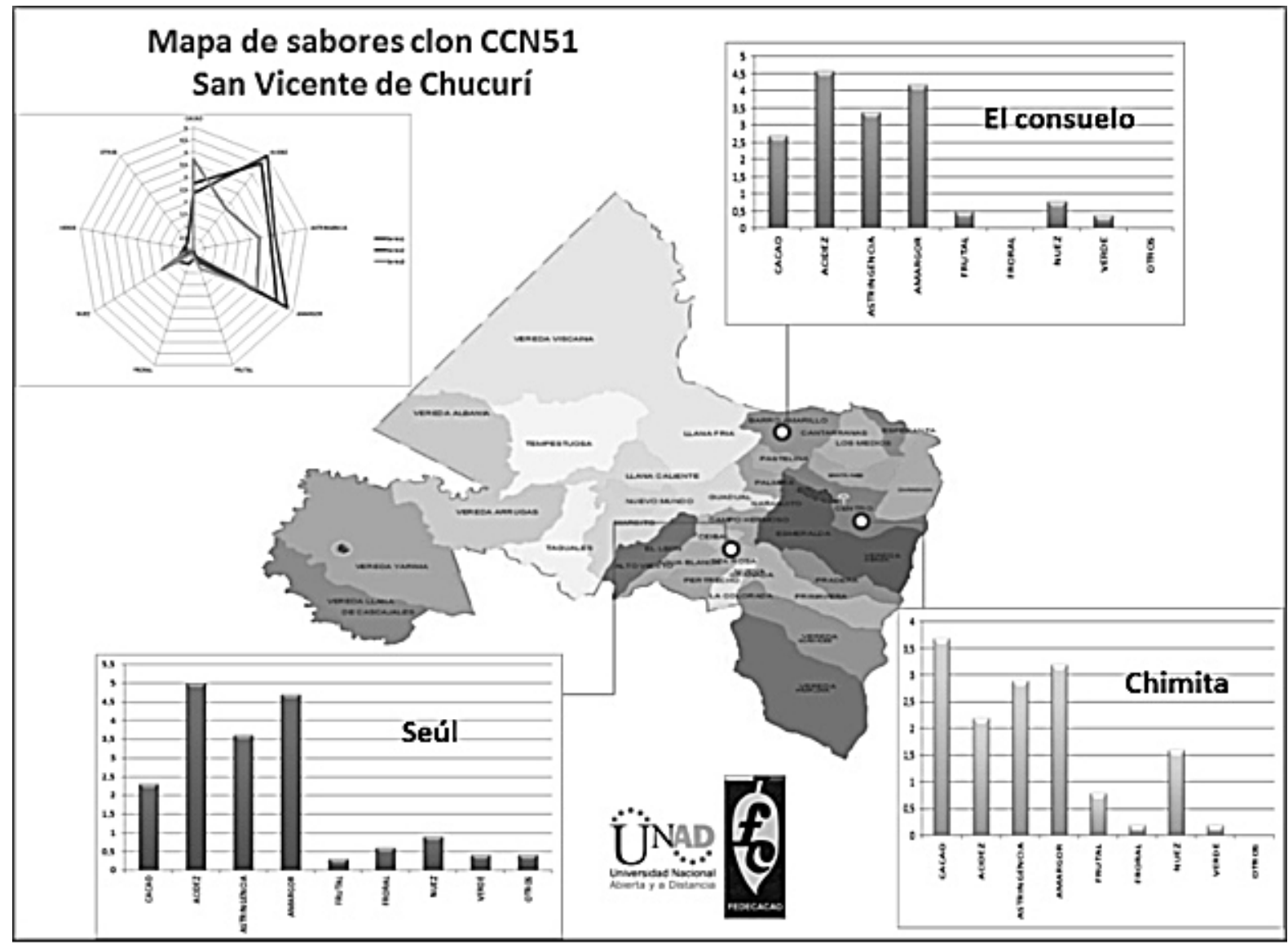

Fuente: los autores.

Figura 9. Mapa sensorial clon CCN 51 de San Vicente de Chucurí 


\section{Conclusiones}

Las diferencias en las condiciones agroecológicas reportadas para cada uno de los grupos de productores de cacao parecen no influir en las características de sabor del cacao CCN51, lo que determinó las diferencias obedece al manejo poscosecha realizado a cada uno de los frutos de cada finca.

A pesar de los tiempos y condiciones similares para la fermentación, es esta operación la que inicialmente influye sobre las características del sabor detectadas por el panel. El nivel de fermentación es un factor importante e influye directamente en los resultados de los atributos finales de sabor a cacao, astringente, ácido, amargo, verde y floral.

Se aprecia una mayor correlación de la muestra fermentada al $80 \%$ de la finca Chimita con el sabor a chocolate y en menor grado con el sabor astringente; en la muestra del $60 \%$ de la finca Seúl existe una correlación media con el sabor a chocolate y astringente; por último el licor del $40 \%$ de la finca El Consuelo está relacionado con el sabor astringente y ácido como producto de una deficiente fermentación.

El perfil sensorial obtenido para el clon CCN51 es consistente con el obtenido en otros países; esto nos ratifica la condición de cacao tipo forastero especial para la elaboración de chocolates, en los perfiles obtenidos se confirma esta apreciación por su predominancia de sabor a chocolate y su baja calificación en sabores adquiridos.

Se presenta un mapa inicial de sabores para el clon CCN51 en el municipio de San Vicente de Chucurí, abriéndose la posibilidad de ampliarlo a otros tipos de clon; el mapa obtenido nos presenta las condiciones actuales de la producción de licor de cacao con los protocolos que se usan en la actualidad.

El análisis sensorial en este caso servirá de herramienta para la propuesta de nuevos o ajustes a las condiciones de proceso pos cosecha, especialmente en la fermentación, secado y tostado, buscando consolidar un perfil de sabor constante que logre posicionar al cacao de la región en un estándar reconocido.

\section{Referencias bibliográficas}

[1] Stone y Sidel (1993)

[2] Corpoica (2000). Caracterización y tipificación de los productores de Cacao del Departamento de Santander. Bucaramanga: Corpoica.

[3] Crespo, E. y Crespo, F. (1997). Cultivo y beneficio del cacao CCN51. Guayaquil: Editorial EI Conejo.

[4] ICONTEC. (2007). GTC 165. Analisis sensorial. Metodología. Guía general . Bogotá D.C.: ICONTEC.

[5] Sancho, J., Bota, E. de Castro. (1999). Introducción al análisis sensorial de los alimentos. Barcelona: Edicions Universitat de Barcelona.

[6] ICTA. (2001). Selección de jueces para evaluación sensorial. En Evaluación sensorial. Bogotá: ICTA.

[7] ICONTEC (1990). NTC 2680. Industrias alimentarias. Análisis sensorial. Ensayo de comparación por pares. Bogotá D.C.: ICONTEC.

[8] ICONTEC (2006). NTC 3883. Análisis sensorial. Metodología. Prueba dúo-trío. Bogotá D.C.: ICONTEC.

[9] ICONTEC. (2008). NTC 2681. Analisis sensorial. Metodologia. Prueba triangular . Bogotá D. C.: ICONTEC.

[10] ICONTEC (1997). NTC 4130. Análisis sensorial. Guía general para la selección, entrenamiento y seguimiento de evaluadores. Parte 2. Expertos. Bogotá D.C.: ICONTEC. 
[11] ICONTEC. (1996). NTC 3884. Analisis sensorial. Guia general para el diseño de cuartos de prueba Bogotá D.C.: ICONTEC.

[12] Ibáñez (2001).

[13] ICONTEC. (2009). GTC 178-2. Análisis sensorial. Guía general para el personal de un laboratorio de evaluacion sensorial parte 2 reclutamienteo y formacion de lideres del panel . Bogotá D.C.: ICONTEC.

[14] Quintana, L y Gómez, S. (2010). Módulo evaluación sensorial. Bucaramanga: UNAD.

[15] Stevenson, C., Corven, J. y., \& G. (1993). Manual para el análisis de cacao en el laboratorio. San José, Costa Rica: IICA.

[16] Gabriela, M. (1985). Evaluación sensorial en el control de calidad de los alimentos procesados. Bo- gotá D.F.: Universidad Nacional.

[17] ICONTEC. (2009). NTC 3929. Analisis sensorial. Metodología. Métodos del perfil del sabor. Bogotá D.C.: ICONTEC.

[18] Jiménez, J., Amores, F., Nicklin, C., Rodriguez, D., \& otros. (2011). Micro fermentación y análisis sensorial para la selección de árboles superiores de cacao. Quevedo: INIAP.

[19] Amores, F., Palacios, A., \& Jimenez, J. y. (2009). Entorno ambiental, genètica, atributos de calidad y singularizaciòn del cacao en el nor oriente de la provincia de esmeraldas. Quevedo: INIAP.

[20] Costell, E. Durán. (1981). El análisis sensorial en el control de calidad de los alimentos. III. Planifica- ción, selección de jueces y diseño estadístico. Instituto de Agroquímica y Tecnología de alimentos. CSIC, 454 - 470.

[21] ACTA. (1997). Taller. Manejo y análisis de datos de evaluación sensorial. Bogotá D.C.: RIEPSA.

[22] ICONTEC. (2001). NTC 4934. Analisis sensorial. Metodología. Guía general para establecer un perfil sensorial. Bogotá D.C.: ICONTEC.

[23] Hernández, R, Fernández, C y Baptista, P (2006). Metodología de la investigación. México D.F.: Mc- Graw Hill.

[24] Marie, P. D. (1996). Evaluación sensorial de los alimentos. Métodos analíticos. México D.F.: Alhambra Mexicana.

[25] ICONTEC. (1997). Analisis sensorial. Guia general para la selección y seguimiento de evaluadores. Parte 1 evaluadores seleccionados. Bogotá D.C.: ICONTEC.

[26] Morales, A. (1994). La evaluación sensorial de los alimentos en la teória y la práctica. Zaragoza: Acribia.

[27] Pedrero, D. (1996). Evaluación sensorial de los alimentos métodos analíticos. México: Alhambra Mexicana.

[28] Rincón. R (2007). Censo cacaotero en Santander. Bucaramanga: FEDECACAO. 